\title{
Novel voice activity detection based on cepstrum moments
}

\begin{abstract}
Statistical methods for voice activity detection (VAD) have shown impressive performance especially with respect to their ability to be tuned parametrically and adoptability with deferent environments. In this paper we propose a novel statistical VAD algorithm using Cepstrum coefficients and their moments as features for classification. In this method, we use moment ratio of conversation part and silent part to evaluate a threshold measure for differentiating between silent and active (Speech) parts of conversation. To make it robust in noisy environments, we will gradually tune the threshold to adopt it with dynamic background noise. Simulation results show that our proposed method has good performance in noisy environments.
\end{abstract}

